\title{
The Prevalence and Treatment Options of Melasma as Well as its Association with Quality of Life in Darker Skin Types: A Scoping Review Protocol
}

Nomakhosi Mpofana ( $\nabla$ mpofanancput@gmail.com )

University of KwaZulu-Natal Nelson R Mandela School of Medicine: University of KwaZulu-Natal College of Health Sciences https://orcid.org/0000-0002-3007-1147

\section{Buyisile Chibi}

University of KwaZulu-Natal College of Health Sciences

Nceba Gqaleni

University of KwaZulu-Natal - Howard College Campus

Ahmed Hussain Mohammed

Cape Technikon: Cape Peninsula University of Technology

\section{Avenal Jane Finlayson}

Durban University of Technology Alan Pittendrigh: Durban University of Technology

\section{Kabelo Kgarosi}

University of Pretoria Department of Library Services: University of Pretoria

\section{Ncoza Cordelia Dlova}

University of KwaZulu-Natal Nelson R Mandela School of Medicine: University of KwaZulu-Natal College of Health Sciences

\section{Protocol}

Keywords: Melasma, chloasma, hyperpigmentation, hypermelanosis, treatment, therapy.

Posted Date: November 15th, 2021

DOI: https://doi.org/10.21203/rs.3.rs-1061210/v1

License: (9) This work is licensed under a Creative Commons Attribution 4.0 International License. Read Full License 


\section{Abstract}

\section{Background}

Melasma is one of the most encountered dermatoses in dermatology and skin care clinics. It is a challenging chronic, recurrent condition associated with hyperpigmentation. Its etiology is poorly understood. Melasma affects all races and gender but is more prevalent in women with darker skin types. Being a facial lesion, melasma has a severe impact on quality of life due to its disfigurement. While many modalities of treatment for melasma exists, unfortunately, effectiveness and safety remain a huge concern. Treatment modalities are variable and often unsatisfactory. The objective of this scoping review is to systemically map available evidence from literature regarding melasma, garner insight as to how melasma affects the quality of life and begin to investigate and gain understanding on effectiveness of different treatments used for melasma.

Methods

A scoping review guided by Arksey and O'Malley's framework, the enhancements and recommendations of Levac, Colquhoun and O'Brien, Daudt and associates and the 2015 Johanna Briggs Institute's guidelines will be conducted. Systematic electronic searches of databases and search engines such as, Scopus, PubMed, CINAHL Complete, Cochrane, Science Direct, and Web of Science will be conducted to attain published peer-reviewed articles of all study designs excluding reviews. All literature that meets the inclusion criteria, research question and sub-question will be included in this review. All the retrieved literature will be exported to an Endnote X20 library. Quality appraisal of the included articles will be conducted using the mixed methods appraisal tool (MMAT) 2018 version.

\section{Discussion}

We anticipate mapping relevant literature on the prevalence of melasma, investigating the effectiveness of treatment options of melasma as well as evaluating its association with quality of life in darker skin types. This study is likely to reveal research gaps, which could guide future implementation research on melasma treatment interventions.

Protocol registration: This protocol has been registered apriori with OSF and is accessible on this link: https://osf.io/ru3jc/

\section{Background}

Melasma remains a huge medical burden. Melasma is one of the most common hyperpigmentation skin disorders seen more frequently in pigmented skin individuals (Black, mixed ancestry, Indians, Hispanics and African Americans) (1-3). In a study conducted in South Africa in the public sector by Dlova et al, 2019 pigmentation disorders are in the top 5 skin conditions seen, with melasma accounting for most of the cases (4). While this skin disorder is common among women, men also get affected $(5,6)$. There is no 
known cure for melasma and currently, available treatment is meant to either halt, stabilize or improve the appearance and progression of the condition (7-9). The current standard treatment for melasma includes the use of hydroquinone which is the standard benchmark and other depigmenting agents like arbutin, kojic acid and retinoids $(6,10-13)$. Some of these therapeutic modalities have side effects like contact dermatitis and ochronosis, especially if used for long periods at higher concentrations without proper monitoring by dermatologists. Due to its complex pathogenesis, melasma is challenging to treat and can be quite disfiguring. There is a convincing body of knowledge showing evidence of a negative impact on the quality of life of those affected (14-18).

Concerns about side effects and the long-term safety of current melasma interventions have spurred efforts to develop alternative treatment options. In rural arrears, black African women use, among others, indigenous plants, for example, Cassipourea flanaganii in an attempt to treat hyperpigmentation disorders (19-22). However, $C$. flanaganii is rapidly becoming extinct as it is highly sought after by the communities $(19,20,22-24)$. It is known that prolonged use of skin lightening products, has been proven to be a significant problem for public health as such use can lead to therapeutic failure and drug resistance which could result in death $(25,26)$. Most of these ingredients are harmful and pose a health risk considering the frequency of application, the duration of practice, the area of the body involved, their use during pregnancy and lactation put their developing foetus or infants at high risk $(27,28)$. Likewise, prolonged use of $C$. flanaganii may pose health risks similar to skin lightening products.

The aim of this scoping review is to map evidence from literature on effects of melasma and glean available information on efficacy of various interventions used to treat melasma on darker skin types. Insights gained from this study will help identify and guide the development of an alternative, safe and cost-effective treatment for melasma, which can be locally manufactured in South Africa. Most therapeutic interventions for melasma are imported, making the costs exorbitant and not accessible to the poor of the poorest, resulting in abuse of unsolicited over the counter banned skin bleaching creams. In addition to commercialisation through cultivation, it is anticipated that knowledge gained will contribute toward the creation of beneficiation for rural communities through the biodiversity act for the indigenous communities, particularly during recent times where unemployment rates are skyrocketing in SA.

\section{Methodology}

The purpose of this scoping review is to systemically map available evidence from literature regarding the prevalence of melasma, garner insight as to how melasma affects the quality of life and begin to investigate and gain an understanding of the treatments used for hypermelanosis. Furthermore, insights gained from this scoping review will enable and generate a deep scientific understanding when dealing with melasma and may help enhance the current melasma treatment armamentarium.

The research question that guides this scoping review is: 
What effect does melasma have on darker skin types and what information is available on efficacy of various treatment interventions?

To answer the question, a scoping review will be conducted. Guided by Arksey and O'Malley's framework (29) enhanced by Levac et al., 2010 (30) and the 2015 Joanna Briggs Institute's guidelines (31), evidence in the literature regarding melasma will be mapped. In accordance with this methodological framework, the systematic search will include the six-stage framework as follows: 1. identifying the research question, 2. identifying relevant studies, 3 . study selection, 4. charting the data, 5. collating, summarizing, and reporting the articles, and 6. consultation (knowledge translation). To ensure no steps are omitted, the Preferred Reporting Items for Systematic Reviews and Meta-Analysis: Extension for Scoping Review guidelines (PRISMA-ScR) will guide the process (32) (Figure 1). Furthermore, the results will be summarized and presented using the Preferred Reporting Items for Systematic Review and Meta-Analysis Protocols (PRISMA-P) 2015 checklist to ensure a rigorous process (33).

A systematic search to synthesise literature for published and unpublished records and articles will be undertaken. The Preferred Reporting Items for systematic Reviews and Meta-analyses (PRISMA) guidelines and the Population, Exposure, and Outcome (PEO) framework for determining the eligibility of the research question (Table 1) will be followed. Since the study will utilise a secondary synthesis of data, ethical approval and consent to participate in the study are not necessary as evidence is already available in the public domain.

\section{Framework stage 1: identifying the research question}

The main research question to guide this scoping review study is:

What effect does melasma have on darker skin types and what information is available on efficacy of various treatment interventions?

Sub research questions are:

1. What is the prevalence of melasma?

2. How does melasma affect the quality of life of people?

3. What treatment interventions are used to treat melasma?

4. Are there any acceptable, safety systematic profile after a long-term use of these treatments?

A Population, Exposure, Outcome (PEO) framework will be applied to adequately address the research question and eligibility of selected and included literature (Table 1). For population, we will include studies done on all people, both male and female, who are classified as darker skin types who suffer from facial melasma. For exposure, we will include studies of treatments used for melasma and for outcome we will look at efficacy of treatment, reported toxicity and a possible improvement in quality of life. Types of treatment interventions used for melasma include medical and non-medical interventions. Studies could be published in any language and will not be limited by time frame to ensure that we cover the 
breadth and comprehensiveness of the available literature. Studies with no evidence of melasma treatment interventions will be excluded as well as studies that investigated extra-facial melasma. The range of evidence will include different methodologies from qualitative to quantitative in nature. Sources such as grey literature and unpublished theses and dissertations will be included.

Table 1

PEO framework

\begin{tabular}{|ll|}
\hline Criteria & Determinants \\
\hline Population & People suffering from melasma \\
\hline Exposure & Any kind of treatment for melasma \\
Outcome & (1) Was the treatment efficient? \\
& (2) reported toxicity or \\
& (3) bad/improved quality of life? \\
\hline
\end{tabular}

\section{Framework stage 2: identifying relevant studies}

Search terms will be applied in a comprehensive electronic literature search which includes the following databases: Systematic electronic search of databases and search engines such as, Scopus, PubMed, Cochrane, CINAHL Complete, ScienceDirect, and Web of Science Core Collection will be conducted to attain published peer-reviewed articles of all study designs excluding reviews. This literature search will not be restricted by language or date of publication. All studies included in the review will be amalgamated and stored using the Endnote $x 20$ library to avoid duplicates in the database (34). We will work closely with the librarians from University of Pretoria and Durban University of Technology during database searching and retrieval of articles. A search strategy using Boolean terms 'AND' and 'OR' to separate search words and terms as well as MeSH terms and keywords will be used. The search results with keywords, search string, Boolean terms, databases and the number of articles retrieved will be recorded on an electronic database search recording table (Table 2). To obtain additional literature, a hand search of included articles reference lists will be conducted.

The search terms will include" melanosis", "Melasma", "skin pigmentation", "dyschromia", "hyperpigmentation", "Chloasma”, "Fitzpatrick skin IV to VI", "Fitzpatrick skin IV - VI", "Fitzpatrick VI", "Fitzpatrick IV", "Black", "Indian”, "Mixed ancestry”, "darker skin type*", "African Continental Ancestry Group", "mixed race","quality of life", "prevalence", "therapy", "therapeutics", "treatment". Boolean terms, AND or "OR" will be used to separate the key words, for example: Melasma OR pigmentation OR dyschromia OR hyperpigmentation OR Chloasma AND "quality of life" OR prevalence OR therapy OR therapeutics OR treatment. Each search term will be documented in detail showing the date of search, search engine, key words used and number of publications retrieved (Table 2).

\section{Framework stage 3: study selection}


Articles and records will be regarded as either eligible for inclusion or exclusion if they meet the following criteria:

\section{Inclusion criteria}

- Articles with evidence of facial melasma

- Articles with evidence of treatment interventions used for facial melasma

- Articles with evidence of prevalence of facial melasma

- Articles with evidence of effects of facial melasma on quality of life

- Peer reviewed articles

- No limitation in terms of time frame

- No limitation in terms of language

\section{Exclusion criteria}

- Articles with no evidence of facial melasma

- Articles with no evidence of treatment interventions used for facial melasma

- Articles with no evidence of prevalence of facial melasma

- Articles with no evidence of effects of facial melasma on quality of life

- Review studies

- Gray literature

We have conducted a pilot search in PubMed in accordance with the inclusion criteria to demonstrate the feasibility of answering our research question using a scoping review method. The results of our pilot search are presented in Table 2. To reduce any selection bias, screening of study titles and abstracts from the databases listed above will be conducted by two investigators independently. The principal investigator will screen titles of all records retrieved from database search and export all eligible records to the endnote library. Using google forms, the two reviewers (primary and secondary authors) will review in parallel all abstracts applying the inclusion criteria to determine eligibility of the selected and identified records. Google forms will also be piloted to ensure all screeners understand how to utilize the tool. Both reviewers will subsequently conduct full-text screening of all eligible records. Discrepancies between the two reviewers will be resolved through discussion and by inviting a third reviewer. To determine the level of agreement between the two reviewers, the kappa statistics will be used (35). A kappa statistic of $>0.21$ will be considered acceptable agreement while $>0.61$ will be considered as an adequate agreement and $0.81-0.99$ will be considered as almost perfect agreement. 
Table 2

Results of Pilot Search in PubMed

\begin{tabular}{|c|c|c|c|}
\hline $\begin{array}{l}\text { Date of } \\
\text { search }\end{array}$ & $\begin{array}{l}\text { Electronic } \\
\text { database }\end{array}$ & Keywords searched & $\begin{array}{l}\text { Number of } \\
\text { publications }\end{array}$ \\
\hline $12 / 10 / 21$ & PubMed & $\begin{array}{l}\text { melanosis [Text Word] OR "skin pigmentation"[MeSH Terms] } \\
\text { OR Skin Pigmentation [Text Word] OR dyschromia [Text } \\
\text { Word] OR "hyperpigmentation"[MeSH Terms] OR } \\
\text { hyperpigmentation [Text Word] OR Chloasma [Text Word] } \\
\text { AND (humans[Filter])) AND (("Quality of Life"[Mesh]) OR } \\
\text { "Prevalence"[Mesh] OR "quality of life"[TW] OR } \\
\text { prevalence[tw] OR "therapy"[Subheading] OR "therapeutics" } \\
\text { MeSH Terms] OR treatment[Text Word] AND (Fitzpatrick } \\
\text { skin IV to VI[TW] OR Fitzpatrick skin IV - VI[TW] OR } \\
\text { Fitzpatrick VI[TW] OR Fitzpatrick IV[TW] OR Black[TW] OR } \\
\text { Indian[TW] OR "mixed ancestry"[TW] OR "darker skin type*" } \\
\text { [TW] OR "African Continental Ancestry Group"[Mesh] OR } \\
\text { "mixed race"[tw] }\end{array}$ & 888 \\
\hline
\end{tabular}

\section{Framework stage 4: charting the data}

Data charting form, will be designed using google forms (Table 3). Data chart form will be independently populated electronically by the screeners with all literature that possesses characteristics and the key information relevant to the review question. The data chart form will be piloted by the two independent reviewers using a random sample of 5 included articles for consistency. The results will be presented as a 'map' of the data in a logical table form that aligns to the research question and scope of the review. During this iterative process the data charting form will be kept current by regularly updating it to ensure accuracy and rigour. 
Table 3

Data charting form

\begin{tabular}{|l|}
\hline Data chart heading \\
\hline Title of study \\
Author and year of publication \\
\hline Study location \\
\hline Study sector/setting \\
\hline Study Aim \\
Study design \\
\hline Study population (Fitzpatrick skin types) \\
\hline Types of data sources included \\
\hline Reported prevalence \\
\hline Reported treatment interventions \\
\hline Reported impact on QoL \\
\hline Reported on clinical features \\
\hline Study findings \\
\hline Reported challenges or limitations \\
\hline Conclusion \\
\hline
\end{tabular}

\section{Framework stage 5: collating, summarizing, and reporting the results}

This process comprises of three stages 1 . descriptive, thematic, and quantitative analysis 2 . reporting the results, and 3. identifying literature gaps for future research. The themes from the extracted data will be examined in relation to the aim of the study which is to map evidence from literature on effects of melasma and glean available information on efficacy of various interventions used to treat melasma on darker skin types. A narrative report will be produced to summarize the extracted data around the following outcomes: interventions used to treat melasma; prevalence of melasma; effects of melasma on quality of life and efficacy. These results will be described in relation to the research question and the context of the overall study purpose. The review results will be published in a peer-reviewed journal and presented at relevant conferences.

\section{Quality appraisal}

To determine the quality of the selected studies, this review will utilize the Mixed Method Appraisal tool (MMAT) version 2018 to appraise all included evidence. This will help avoid any risk of bias and also 
ensure that all evidence included in the study is appropriate (36). The MMAT is a critical appraisal tool designed for the appraisal stage of systematic mixed studies reviews, for example, reviews that include both quantitative and qualitative as well and mixed methods studies (36). According to Hong et al (2018), the MMAT tool permits appraisal of the methodological quality of five categories of studies as follows: 1 . qualitative research, 2 . randomized controlled trials, 3. non-randomized studies, 4 . quantitative descriptive studies, and lastly 5 . mixed methods studies. The two screeners will be responsible for assigning ratings such as $100 \%$ for high average articles, $75 \%$ for above-average articles, $50 \%$ average and $25 \%$ for lowquality articles.

\section{Discussion}

This scoping review aims to identify and describe the effects of melasma on those who suffer from it by systematically mapping the evidence available in literature, garner insight on its prevalence and how melasma affects the quality of life and to investigate and gain an understanding of the efficacy of different interventions used for hypermelanosis on darker skin types. Numerous treatment interventions such as hydroquinone (HQ), kojic acid, arbutin, chemical peels, micro-needling, lasers and lights are used to manage melasma, however the effectiveness of these treatment interventions is limited due to their adverse effects $(6,10-13)$. Currently, there is no cure for melasma and treatment is meant to be chronic to try and minimize progression. Being a facial disfiguring lesion, many authors have published data showing evidence of a negative impact on the quality of life of those affected by the condition (7-9). In rural areas women use plants including $c$. flanaganii in an attempt to resolve hypermelanosis, however the plant is becoming extinct due to over harvesting $(19,20,22-24)$, also long-term systematic effects due to prolonged use of this plant are not yet known.

Melasma is reported to be a huge medical burden. It has been highly reported in patients of Hispanic, African American, Arab, South East Asian, East Asian and African descents (1-3). Despite numerous attempts to resolve the problem, it is evident that the problem still exists. While many modalities of treatment for melasma exist, unfortunately effectiveness and safety remain a huge concern. Treatment modalities are variable and often unsatisfactory. This portrays a lack of ideas when optimal relief cannot be offered.

The insights gained from this proposed scoping review could help enhance the scientific understanding on how to address melasma. Insights gained from the study could assist in commercialisation and in addition create beneficiation for rural communities. Possible outcomes for this study may include the identification of a holistic, safe and cost effective alternative and desirable treatment plan for melasma which can be locally manufactured in South Africa, therefore adding to the current treatment melasma armamentarium and in turn improving the quality of life. We plan to disseminate the study's findings in peer-reviewed journals and at conferences that focus on dealing with hypermelanosis and present at both international and national seminars. This study could assist in mapping out literature gaps pertaining to treatment of melasma in darker skin types. The results will help offer recommendations on how further research can address and close these gaps. 


\section{Limitations}

The study results will be limited by skin types as it only focuses on darker skin types. Strict time lines will be adhered to.

\section{Abbreviations}

HQ: Hydroquinone; C. flanaganii: Cassipourea flanaganii; SA; South Africa; WHO: World Health Organisation; PRISMA ScR: Preferred Reporting Items for Systematic Reviews and Meta-Analysis: Extension for Scoping Reviews; PEO: Population Exposure Outcome; MeSH: Medical Subject Heading; MMAT: Mixed Method Appraisal Tool; UP: University of Pretoria.

\section{Declarations}

\section{Supplementary data}

PRISMA ScR

Waiver Request Letter

\section{Acknowledgments}

We wish to acknowledge Dr Desmond Kuupiel for his professional advice on this protocol. The corresponding author wishes to express sincere gratitude to Durban University of Technology for enrolling her in the UP-mentorship programme.

\section{Authors contributions}

Concept and design, NM, BC, Data base search: KK, AF; writing of the manuscript: NM; review and editing: BC, NCD; AHM, NG. All authors have reviewed and approved the final drafted manuscript.

\section{Funding}

Not Applicable

\section{Availability of data and materials}

All data generated or analysed during this study will be included in the published scoping review article and will be available upon request.

\section{Ethics approval and consent to participate}

Not applicable

Consent for publication 
Not applicable

\section{Competing interests}

The authors declare that they have no competing interests.

\section{References}

1. D’Elia MPB, Brandão MC, de Andrade Ramos BR, da Silva MG, Miot LDB, Dos Santos SEB, et al. African ancestry is associated with facial melasma in women: a cross-sectional study. BMC Med Genet. 2017;18(1):1-7.

2. Gupta S, Mahendra A, Jindal A, Kaur G. A study of the clinical profile and assessment of the quality of life in patients of Melasma. Pharma Innov. 2017;6(7, Part C):190.

3. Qazi I, Dogra N, Dogra D. Melasma: a clinical and epidemiological study. Int J Contemporary Med Res. 2017;4(10):2088-90.

4. Dlova NC, Akintilo LO, Taylor SC. Prevalence of pigmentary disorders: A cross-sectional study in public hospitals in Durban, South Africa. Int J Womens Dermatol. 2019;5(5):345-8.

5. Rostami Mogaddam M, safavi Ardabili N, Iranparvar Alamdari M, Maleki N, Aghabalaei Danesh M. Evaluation of the serum zinc level in adult patients with melasma: Is there a relationship with serum zinc deficiency and melasma? J Cosmet Dermatol. 2018;17(3):417-22.

6. Sarkar R, Arsiwala S, Dubey N, Sonthalia S, Das A, Arya L, et al. Chemical peels in melasma: A review with consensus recommendations by Indian pigmentary expert group. Indian J Dermatol. 2017;62(6):578.

7. Dunbar S, Posnick D, Bloom B, Elias C, Zito P, Goldberg DJ. Energy-based device treatment of melasma: An update and review of the literature. J Cosmet Laser Ther. 2017;19(1):2-12.

8. Grimes P, ljaz S, Nashawati R, Kwak D. New oral and topical approaches for the treatment of melasma. Int J Womens Dermatol. 2019;5(1):30-6.

9. Rafi S, Iftikhar U, Rani Z, Hussain I. Comparison of efficacy and safety of topical hydroquinone $2 \%$ and oral tranexamic acid $500 \mathrm{mg}$ in patients of melasma. Journal of Pakistan Association of Dermatology. 2018;27(3):204-13.

10. Chalermchai T, Rummaneethorn P. Effects of a fractional picosecond 1,064 $\mathrm{nm}$ laser for the treatment of dermal and mixed type melasma. J Cosmet Laser Ther. 2018;20(3):134-9.

11. Cheng J, Vashi NA. Chemical peels in ethnic skin. In: Vashi NA, Maibach H, editors. Dermatoanthropology of ethnic skin and hair. Springer; 2017. p. 437-48.

12. Handel AC, Miot LDB, Miot HA. Cosmetic approach for melasma. In: Issa MCA, Tamura B, editors. Daily Routine in Cosmetic Dermatology. Springer; 2017. p. 419-32.

13. Nomakhosi M, Heidi A. Natural options for management of melasma, a review. J Cosmet Laser Ther. 2018;20(7-8):470-81. 
14. Arora P, Meena N, Sharma P, Raihan M. Impact of melasma on quality of life in Indian patients. Pigment Int. 2017;4(2):92.

15. Cohen PR. Melasma treatment: A novel approach using a topical agent that contains an antiestrogen and a vascular endothelial growth factor inhibitor. Med Hypotheses. 2017;101:1-5.

16. Jiang J, Akinseye O, Tovar-Garza A, Pandya A. The effect of melasma on self-esteem: A pilot study. Int J Womens Dermatol. 2018;4(1):38-42.

17. Mpofana $\mathrm{N}$, Abrahamse $\mathrm{H}$. The management of melasma on skin types $\mathrm{V}$ and $\mathrm{VI}$ using light emitting diode treatment. Photomed Laser Surg. 2018;36(10):522-9.

18. Uyanikoglu H, Aksoy M. Quality of life in patients with melasma in Turkish women. Dermatol Reports. 2017;9(2):7340.

19. Afolayan AJ, Grierson DS, Mbeng WO. Ethnobotanical survey of medicinal plants used in the management of skin disorders among the Xhosa communities of the Amathole District, Eastern Cape, South Africa. J Ethnopharmacol. 2014;153(1):220-32.

20. Bhat R. Plants of Xhosa people in the Transkei region of Eastern Cape (South Africa) with major pharmacological and therapeutic properties. J Med Plant Res. 2013;7(20):1474-80.

21. Dlova NC, Ollengo MA. Traditional and ethnobotanical dermatology practices in Africa. Clin Dermatol. 2018;36(3):353-62.

22. Thibane V, Ndhlala A, Abdelgadir H, Finnie J, Van Staden J. The cosmetic potential of plants from the Eastern Cape Province traditionally used for skincare and beauty. S Afr J Bot. 2019;122:475-83.

23. Langat MK, Dlova NC, Mulcahy-Ryan LE, Schwikkard SL, Opara El, Crouch NR, et al. The effect of isolates from Cassipourea flanaganii (Schinz) alston, a plant used as a skin lightning agent, on melanin production and tyrosinase inhibition. J Ethnopharmacol. 2021;264:113272.

24. Thibane V, Ndhlala A, Finnie J, Van Staden J. Modulation of the enzyme activity of secretory phospholipase A2, lipoxygenase and cyclooxygenase involved in inflammation and disease by extracts from some medicinal plants used for skincare and beauty. S Afr J Bot. 2019;120:198-203.

25. Browne F, Wilkinson SM. Effective prescribing in steroid allergy: controversies and cross-reactions. Clin Dermatol. 2011;29(3):287-94.

26. Kamm GL, Hagmeyer KO. Allergic-type reactions to corticosteroids. Ann Pharmacother. 1999;33(4):451-60.

27. Bose-O'Reilly S, McCarty KM, Steckling N, Lettmeier B. Mercury exposure and children's health. Curr Probl Pediatr Adolesc Health Care. 2010;40(8):186-215.

28. Mahé E, Girszyn N, Hadj-Rabia S, Bodemer C, Hamel-Teillac D, De Prost Y. Subcutaneous fat necrosis of the newborn: a systematic evaluation of risk factors, clinical manifestations, complications and outcome of 16 children. Bri J Dermatol. 2007;156(4):709-15.

29. Arksey H, O'Malley L. Scoping studies: towards a methodological framework. Int J Soc Res Methodol. 2005;8(1):19-32. 
30. Levac D, Colquhoun H, O'Brien KK. Scoping studies: advancing the methodology. Implement Sci. 2010;5(1):1-9.

31. Peters MD, Godfrey CM, Khalil H, Mclnerney P, Parker D, Soares CB. Guidance for conducting systematic scoping reviews. JBI Evid Implement. 2015;13(3):141-6.

32. Page MJ, McKenzie JE, Bossuyt PM, Boutron I, Hoffmann TC, Mulrow CD, et al. The PRISMA 2020 statement: an updated guideline for reporting systematic reviews. BMJ. 2021;372.

33. Moher D, Liberati A, Tetzlaff J, Altman DG. Preferred reporting items for systematic reviews and meta-analyses: the PRISMA statement. Int J Surg. 2010;8(5):336-41.

34. Hupe M. EndNote X9. J Electron Resour Medical Libr. 2019;16(3-4):117-9.

35. Malpica A, Matisic JP, Van Niekirk D, Crum CP, Staerkel GA, Yamal J-M, et al. Kappa statistics to measure interrater and intrarater agreement for 1790 cervical biopsy specimens among twelve pathologists: qualitative histopathologic analysis and methodologic issues. Gynecol Oncol. 2005;99(3):S38-S52.

36. Hong QN, Fàbregues S, Bartlett G, Boardman F, Cargo M, Dagenais P, et al. The Mixed Methods Appraisal Tool (MMAT) version 2018 for information professionals and researchers. Educ Inf. 2018;34(4):285-91.

\section{Figures}




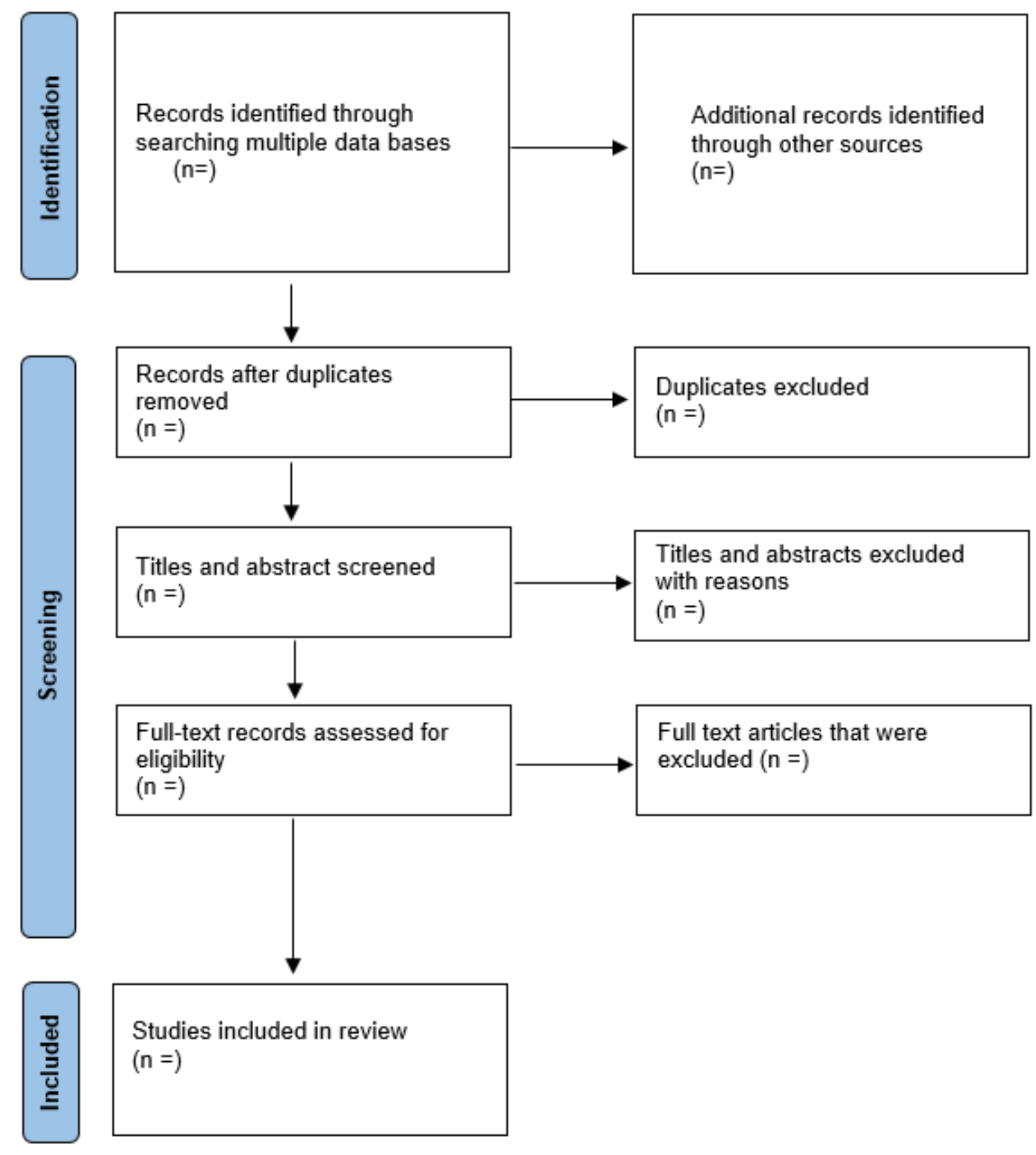

Figure 1

PRISMA-ScR flow diagram demonstrates the literature search and study selection process (32).

\section{Supplementary Files}

This is a list of supplementary files associated with this preprint. Click to download.

- BMCSystematicReviewsPRISMAScR.pdf

- BMCSYSTEMATICREVIEWSWAIVERREQUESTLETTER.pdf 